\title{
WAIST CIRCUMFERENCE-TO-HEIGHT RATIO DETECTED IN A CONVENIENT SAMPLE OF YOUNG SLOVAK PEOPLE WITH INCREASED CARDIO-METABOLIC RISK
}

\author{
Zdenka Hertelyová1, Janka Vašková2, Ladislav Vaško² \\ 'Department of Experimental Medicine, Faculty of Medicine, Pavol Jozef Šafárik University in Košice, Košice, Slovak Republic \\ ${ }^{2}$ Department of Medical and Clinical Biochemistry, Faculty of Medicine, Pavol Jozef Šafárik University in Košice, Košice, Slovak Republic
}

\section{SUMMARY}

Background and Aim: Cardiovascular disease is a serious problem increasingly affecting young people worldwide. Cardio-metabolic risk factors include lipid profile parameters and atherogenic index of plasma (AIP), as well as basic anthropometric parameters such as the ratio of waist circumference to height (WHtR). The aim of our study was to determine WHtR.

Method: WHtR was determined in a group of 500 clinically healthy young people aged 18-25 and, on the basis of measured data, a reference range for WHtR was designed.

Results: The average WHtR value for the whole group was $0.45 \pm 0.06$, with $0.46 \pm 0.06$ for men, and $0.44 \pm 0.06$ for women. WHtR values in respondents with all other parameters in normal range were from 0.41 to 0.52 for men and from 0.38 to 0.50 for women. Values are similar to those observed in other studies across diverse world populations. A positive correlation was observed between WHtR and atherogenic indices (AP1, AIP) uric acid levels and lipid profile parameters at $p<0.001$, with the exception of glucose and HDL. WHtR and HDL were negatively correlated at $p<0.001$.

Conclusion: As WHtR can be easily determined, it can be used as another cardio-metabolic risk factor.

Key words: atherogenic parameters, cardio-metabolic risk, lipid profile, waist circumference to height ratio, young people

Address for correspondence: L. Vaško, Department of Medical and Clinical Biochemistry, Faculty of Medicine, Pavol Jozef Šafárik University in Košice, Tr. SNP 1, 04066 Košice, Slovak Republic. E-mail: ladislav.vasko@upjs.sk

http://dx.doi.org/10.21101/cejph.a4007

\section{INTRODUCTION}

In most Central and Eastern European countries, cardiovascular disease mortality increased during the 1970 s and 1980 s and started to decline in the early to mid-1990s (1,2). Despite the recent decrease, mortality rates are still considerably higher when compared with Western European countries. Although most Central and Eastern European countries appear to have reached their peak in cardiovascular mortality, the majority can still be clearly classified as high-risk countries (2). Slovakia is geographically situated in Central Europe and is therefore affected by this trend. Remarkably, cardio-metabolic risk factors are already emerging at an increasingly younger age $(3,4)$.

Advances in the prevention and treatment of cardio-metabolic risk factors have led to a significant reduction in related mortality. Such risk factors are divided by origin into external and internal, and by manageability into modifiable and unmodifiable. The major modifiable risk factors are high levels of TC and/or LDL in blood, TAG, low HDL, cigarette smoking, type 2 diabetes mellitus (DM), and arterial hypertension. The secondary modifiable risk factors include visceral obesity, insulin resistance, lack of exercise coupled with a sedentary lifestyle, unbalanced diet composition, types of psychosomatosis, stress (psychosocial burden), and also sudden hormonal changes (5). In particular, it should be mentioned that the controversial role of serum uric acid levels (antioxidant properties versus NO inactivation, lipid peroxidation and the formation of reactive intermediates such as alkylating species), makes this parameter a potential metabolic risk factor (6).

While the determination of total cholesterol (TC), serum triglyceride (TG), high-density lipoprotein cholesterol (HDL), low-density lipoprotein (LDL) cholesterol, and blood glucose form part of the basic biochemical laboratory tests, there are however concerns about the reliability of some anthropometric measurements (7). Body mass index (BMI) has routinely been used in clinical and public health practice to identify individuals at risk of future cardiovascular disease and diabetes. However, this measurement reflects both fat and lean mass and does not identify fat distribution (8). Other parameters usually used to assess obesity and abdominal obesity in particular, such as waist circumference (WC) and hip circumference (HC), may differ depending on the precise site at which they are measured (9).

In the last few years, several large studies throughout different populations have shown that waist circumference-to-height ratio (WHtR) correlates more strongly with cardio-metabolic 
risk factors and is a simple and effective anthropometric index to identify obesity-associated metabolic risks. The optimal cut-off values for WHtR in the detection of cardio-metabolic risk factors were identified by receiver operating characteristic curve analysis confirming value of 0.5 as boundary of Caucasian, Asian and Central American subjects $(10,11)$, in sub-population of ethnic South Asian adults (12), and Chinese adults $(13,14)$.

Because some symptoms indicative of possible risk of cardiovascular disease manifest at younger age, the aim of our study was to investigate the current state of WHtR in a group of clinically healthy young Slovak people. Subsequently, we followed the correlation between WHtR and other cardiovascular risk factors to find out about and provide information on current status and potential health risks for the young population of Slovakia given the current lifestyle, food habits etc.

\section{MATERIALS AND METHODS}

The study was approved by the University Ethics Committee No. 6/2012. A personal interview protocol has been developed on the basis of questions about current health status answered by potential probands. The convenient sample of participants, who at the time of the study felt physical and mental well-being, was identified and included into study as a healthy group. Those suffering from cardiovascular diseases, myocardial infarction, atherosclerosis, stroke, hypertension, gout, diabetes mellitus type 2, familial hypercholesterolemia, ischaemic disease of the lower limbs, varicose veins, or reduced diet and smokers were not included in the study. In addition, pregnant women were also not included.

In total the study group comprised 500 participants, including 150 men (30\%) at an average age of $22.54 \pm 2.54$, and 350 women $(70 \%)$ at an average age of $21.93 \pm 2.41$. The probands were young people (aged 18-25 years), either university students or workers employed in Košice. The vast majority of students were from the Faculty of Medicine in Košice. Since participation in the study was voluntary, the number of participants with respect to gender and background was not subject to any selection and the number of examined respondents with regard to the above criteria therefore led to unbalanced numbers of men and women. Participation in the study was possible between the years 2012-2014, when $10 \mathrm{ml}$ of venous blood was withdrawn from probands from the median cubital vein for biochemical investigation, and anthropometric parameters (weight, height, waist circumference) were measured directly. The blood was left to clot and serum was obtained after centrifugation at 3,500 rpm for 7 minutes. The sera were stored at $-80{ }^{\circ} \mathrm{C}$ and analyzed within one month of collection. A lipid profile (TC, HDL, very-low density lipoprotein (VLDL) cholesterol and TG), uric acid and glucose were determined from blood serum by an enzymatic spectrophotometric method through diagnostic reagents from BioVendor-Laboratory Medicine (Brno, Czech Republic) for the COBAS MIRA biochemical analyzer (Roche, Bern, Switzerland). As previously mentioned by Hertelyová et al. (15), the reference values of selected parameters by the supplier of diagnostic kits were provided. Other lipid parameters were determined, such as non-HDL, LDL by the calculations: non-HDL $=\mathrm{TC}-\mathrm{HDL} ; \mathrm{LDL}$ by equation $=\mathrm{TC}-\mathrm{HDL}-(\mathrm{TG} / 2.2)$ originally described by Friedewald et al. (16). Atherogenic indices, such as AIP and AT1 were calculated as follows: AIP $=\log ($ TAG/HDL $)$ (17), AT1 = LDL/HDL (18).

Results were expressed as mean \pm standard deviation. Significant differences among groups (men, women and total respondents) were determined using the Tukey test (MINITAB for Windows 11.21). Values of $\mathrm{p}<0.05$ were considered significant. Pearson's correlation coefficient was used to estimate the relationships between selected parameters (SPSS version 21.0). The significance of Pearson correlation was determined with Student's t-test (Microsoft Excel 2013). Differences were considered significant at $\mathrm{p}<0.05$.

Table 1. Serum lipid, atherogenic and anthropometric parameters and indices measured in a group $(N=500)$ of healthy young men and women from Slovakia. Statistical significance between men and women by one-way ANOVA

\begin{tabular}{|c|c|c|c|c|}
\hline Parameter & $\begin{array}{c}\text { Men } \\
\text { (mean } \pm \text { SD) }\end{array}$ & $\begin{array}{c}\text { Women } \\
\text { (mean } \pm \text { SD) }\end{array}$ & $p$ & Significance \\
\hline $\mathrm{TC}\left(\mathrm{mmol} . \mathrm{I}^{-1}\right)$ & $4.40 \pm 0.83$ & $4.64 \pm 0.93$ & 0.006 & ** \\
\hline $\mathrm{TG}\left(\mathrm{mmol} . \mathrm{I}^{-1}\right)$ & $1.05 \pm 0.52$ & $0.95 \pm 0.60$ & 0.086 & ns \\
\hline $\mathrm{HDL}\left(\mathrm{mmol} . \mathrm{I}^{-1}\right)$ & $1.12 \pm 0.28$ & $1.44 \pm 0.39$ & $<0.001$ & $* * *$ \\
\hline $\mathrm{LDL}\left(\mathrm{mmol} . \mathrm{I}^{-1}\right)$ & $2.80 \pm 0.73$ & $2.77 \pm 0.79$ & 0.658 & ns \\
\hline VLDL (mmol..$\left.^{-1}\right)$ & $0.41 \pm 0.15$ & $0.39 \pm 0.14$ & 0.084 & ns \\
\hline Non-HDL (mmol..$\left.^{-1}\right)$ & $3.28 \pm 0.81$ & $3.20 \pm 0.87$ & 0.354 & ns \\
\hline $\mathrm{UA}\left(\mathrm{mmol} \mathrm{I}^{-1}\right)$ & $342.19 \pm 66.87$ & $254.26 \pm 60.17$ & $<0.001$ & $* * *$ \\
\hline Glucose (mmol..$\left.^{-1}\right)$ & $4.46 \pm 0.72$ & $4.50 \pm 0.58$ & 0.461 & ns \\
\hline AT1 (mmol..$\left.^{-1}\right)$ & $2.67 \pm 0.96$ & $2.08 \pm 0.88$ & $<0.001$ & $* * *$ \\
\hline AIP & $-0.06 \pm 0.25$ & $-0.22 \pm 0.22$ & $<0.001$ & $* * *$ \\
\hline Weight (kg) & $77.91 \pm 13.47$ & $61.00 \pm 10.78$ & $<0.001$ & $* * *$ \\
\hline Height (cm) & $180.76 \pm 8.22$ & $166.47 \pm 5.75$ & $<0.001$ & $* * *$ \\
\hline WHtR & $0.46 \pm 0.06$ & $0.44 \pm 0.06$ & 0.001 & ** \\
\hline
\end{tabular}

${ }^{* *} \mathrm{p}<0.01,{ }^{* * *} \mathrm{p}<0.001, \mathrm{~ns}-$ not significant 
Table 2. Serum lipid parameters measured in a group $(N=150)$ of healthy young men from Slovakia. Values are separated by the range of values specified by the diagnostic kit

\begin{tabular}{|c|c|c|c|c|c|}
\hline \multicolumn{2}{|l|}{ Parameter } & Low & Normal & Increase & High \\
\hline \multirow{3}{*}{ TC } & $\mathrm{N}$ & 21 & 103 & 22 & 4 \\
\hline & $\%$ & 14 & 68.7 & 14.7 & 2.6 \\
\hline & mmol..$^{-1}$ & $3.17 \pm 0.27^{d}$ & $4.30 \pm 0.42^{c}$ & $5.66 \pm 0.26^{b}$ & $6.37 \pm 0.19^{a}$ \\
\hline \multirow{3}{*}{ TG } & $\mathrm{N}$ & 3 & 126 & - & 21 \\
\hline & $\%$ & 2 & 84 & - & 14 \\
\hline & mmol..$^{-1}$ & $0.45 \pm 0.01^{c}$ & $0.89 \pm 0.30^{b}$ & - & $2.08 \pm 0.28^{a}$ \\
\hline \multirow{3}{*}{ HDL } & $\mathrm{N}$ & 56 & 94 & - & - \\
\hline & $\%$ & 37.3 & 62.7 & - & - \\
\hline & mmol..$^{-1}$ & $0.86 \pm 0.10^{b}$ & $1.27 \pm 0.24^{\mathrm{a}}$ & - & - \\
\hline \multirow{3}{*}{ LDL } & $\mathrm{N}$ & - & 119 & 24 & 7 \\
\hline & $\%$ & - & 79.3 & 16 & 4.7 \\
\hline & mmol..$^{-1}$ & - & $2.51 \pm 0.48^{c}$ & $3.76 \pm 0.23^{b}$ & $4.42 \pm 0.16^{a}$ \\
\hline \multirow{3}{*}{ VLDL } & $\mathrm{N}$ & - & 129 & - & 21 \\
\hline & $\%$ & - & 86 & - & 14 \\
\hline & mmol..$^{-1}$ & - & $0.40 \pm 0.13^{b}$ & - & $0.48 \pm 0.24^{a}$ \\
\hline \multirow{3}{*}{ Non-HDL } & $\mathrm{N}$ & - & 115 & - & 35 \\
\hline & $\%$ & - & 77 & - & 23 \\
\hline & $\mathrm{mmol.I^{-1 }}$ & - & $2.92 \pm 0.51^{b}$ & - & $4.45 \pm 0.43^{\mathrm{a}}$ \\
\hline \multirow{3}{*}{ UA } & $\mathrm{N}$ & - & 131 & - & 19 \\
\hline & $\%$ & - & 87.3 & - & 12.7 \\
\hline & mmol..$^{-1}$ & - & $325.31 \pm 50.10^{b}$ & - & $458.63 \pm 50.00^{a}$ \\
\hline \multirow{3}{*}{ Glucose } & $\mathrm{N}$ & - & 141 & - & 9 \\
\hline & $\%$ & - & 94 & - & 6 \\
\hline & $\mathrm{mmol}^{-I^{-1}}$ & - & $4.51 \pm 0.67^{b}$ & - & $5.82 \pm 0.23^{\mathrm{a}}$ \\
\hline \multirow{3}{*}{ AT1 } & $\mathrm{N}$ & - & 117 & - & 33 \\
\hline & $\%$ & - & 76.7 & - & 23.3 \\
\hline & mmol..$^{-1}$ & - & $2.26 \pm 0.59^{b}$ & - & $4.11 \pm 0.54^{a}$ \\
\hline \multirow{3}{*}{ AIP } & $\mathrm{N}$ & - & 116 & 14 & 20 \\
\hline & $\%$ & - & 77.3 & 9.3 & 13.4 \\
\hline & $\mathrm{mmol} . \mathrm{I}^{-1}$ & - & $-0.16 \pm 0.16^{c}$ & $0.15 \pm 0.03^{b}$ & $0.39 \pm 0.10^{a}$ \\
\hline
\end{tabular}

$a, b, c, d$ values represent statistically significant differences between groups at $p<0.05$ (Tukey test).

\section{RESULTS}

The average height of the entire group was $171.15 \pm 9.14 \mathrm{~cm}$; males $180.76 \pm 8.22 \mathrm{~cm}$ and females $166.47 \pm 5.75 \mathrm{~cm}$ (Table 1$)$. The average waist circumference was $76.22 \pm 11.40 \mathrm{~cm}$; males $83.69 \pm 10.47 \mathrm{~cm}$ and females $74.01 \pm 10.12 \mathrm{~cm}$. The average value of WHtR in the whole group was $0.45 \pm 0.06$; males $0.46 \pm 0.06$ and females $0.45 \pm 0.06$. WHtR values were divided into three groups according to the frequency of occurrence: 1: up to 0.51 , found in 423 subjects $(84.6 \%)$; 2: between 0.51 and 0.63 , found in 71 individuals (14.2\%); and 3 : greater than 0.63 , found in only 6 probands $(1.2 \%)$. In the second group, there was a total of 28 men $(12.2 \%)$ and 43 women $(12.3 \%)$. The third group consisted of 2 men $(1.3 \%)$ and 4 women (1.1\%). 52 subjects had WHtR values less than $0.39(6.67 \%)$, including 5 men $(3.3 \%)$ and 47 women $(13.4 \%)$
Out of 500 respondents, 339 (67.8\%) had physiological levels of TC (3.51 to $5.19 \mathrm{mmol} / \mathrm{l})$, including 103 males $(68.7 \%)$ with an average value of $4.30 \pm 0.42 \mathrm{mmol}^{-1}$, and 236 women $(67.4 \%)$ with an average TC level of $4.39 \pm 0.45 \mathrm{mmol}^{-1} \mathrm{l}^{-1}$ (Table $2,3)$. Low cholesterol levels $\left(\leq 3.5 \mathrm{mmol}^{-1} \mathrm{l}^{-1}\right)$ were observed in 53 cases $(10.6 \%)$, including 21 males $(14.0 \%)$ with a mean value of $3.17 \pm 0.27 \mathrm{mmol}^{-1} \mathrm{l}^{-1}$ and 32 women $(9.1 \%)$ with an average TC level of $3.21 \pm 0.25 \mathrm{mmol} . \mathrm{l}^{-1}$. Increased values of TC (from 5.2 to $\left.6.19 \mathrm{mmol}^{-\mathrm{l}^{-1}}\right)$ were observed in 77 cases $(15.4 \%)$, including 22 men $(14.7 \%)$ with a mean TC value of $5.66 \pm 0.26 \mathrm{mmol}^{-1}$ and 55 women $(15.7 \%)$ with an average level of $5.52 \pm 0.28$ mmol. $\mathrm{l}^{-1}$. High levels of TC $\left(\geq 6.2 \mathrm{mmol} . \mathrm{l}^{-1}\right)$ were measured in 31 subjects $(6.2 \%)$, including 4 males $(2.6 \%)$ with a mean value of $6.37 \pm 0.19 \mathrm{mmol} . \mathrm{l}^{-1}$, and 27 females $(7.8 \%)$ with an average TC level of $6.72 \pm 0.47 \mathrm{mmol}^{-1}$. Differences between groups (in men, women, and all respondents) were statistically significant 
Table 3. Serum lipid parameters measured in a group $(N=350)$ of healthy young women from Slovakia. Values are separated by the range of values specified by the diagnostic kit

\begin{tabular}{|c|c|c|c|c|c|}
\hline \multicolumn{2}{|l|}{ Parameter } & Low & Normal & Increase & High \\
\hline \multirow{3}{*}{ TC } & $\mathrm{N}$ & 32 & 236 & 55 & 27 \\
\hline & $\%$ & 9.1 & 67.4 & 15.7 & 7.8 \\
\hline & $\mathrm{mmol}^{-\mathrm{I}^{-1}}$ & $3.21 \pm 0.25^{d}$ & $4.39 \pm 0.45^{\circ}$ & $5.52 \pm 0.28^{b}$ & $6.72 \pm 0.47^{a}$ \\
\hline \multirow{3}{*}{ TG } & $\mathrm{N}$ & 18 & 303 & - & 29 \\
\hline & $\%$ & 5.2 & 86.6 & - & 8.2 \\
\hline & mmol..$^{-1}$ & $0.38 \pm 0.05^{c}$ & $0.84 \pm 0.30^{b}$ & - & $2.44 \pm 0.93^{a}$ \\
\hline \multirow{3}{*}{ HDL } & $\mathrm{N}$ & 145 & 205 & - & - \\
\hline & $\%$ & 41.4 & 58.6 & - & - \\
\hline & mmol..$^{-1}$ & $1.10 \pm 0.13^{b}$ & $1.68 \pm 0.33^{\mathrm{a}}$ & - & - \\
\hline \multirow{3}{*}{ LDL } & $\mathrm{N}$ & - & 285 & 46 & 19 \\
\hline & $\%$ & - & 81.4 & 13.2 & 5.4 \\
\hline & mmol..$^{-1}$ & - & $2.49 \pm 0.51^{c}$ & $3.67 \pm 0.18^{b}$ & $4.79 \pm 0.52^{\mathrm{a}}$ \\
\hline \multirow{3}{*}{ VLDL } & $\mathrm{N}$ & - & 321 & - & 29 \\
\hline & $\%$ & - & 91.4 & - & 8.2 \\
\hline & $\mathrm{mmol.}^{-1}$ & - & $0.38 \pm 0.13^{b}$ & - & $0.43 \pm 0.27^{a}$ \\
\hline \multirow{3}{*}{ Non-HDL } & $\mathrm{N}$ & - & 282 & - & 68 \\
\hline & $\%$ & - & 80.6 & - & 19.4 \\
\hline & mmol..$^{-1}$ & - & $2.88 \pm 0.54^{b}$ & - & $4.54 \pm 0.72^{\mathrm{a}}$ \\
\hline \multirow{3}{*}{ UA } & $\mathrm{N}$ & - & 336 & - & 14 \\
\hline & $\%$ & - & 96 & - & 4 \\
\hline & mmol..$^{-1}$ & - & $247.87 \pm 49.32^{b}$ & - & $449.64 \pm 71.06^{a}$ \\
\hline \multirow{3}{*}{ Glucose } & $\mathrm{N}$ & - & 344 & - & 6 \\
\hline & $\%$ & - & 98.29 & - & 1.71 \\
\hline & mmol..$^{-1}$ & - & $4.60 \pm 0.52^{b}$ & - & $6.59 \pm 0.97^{\mathrm{a}}$ \\
\hline \multirow{3}{*}{ AT1 } & $\mathrm{N}$ & - & 325 & - & 25 \\
\hline & $\%$ & - & 92.9 & - & 7.1 \\
\hline & mmol..$^{-1}$ & - & $1.92 \pm 0.66^{b}$ & - & $4.18 \pm 0.62^{\mathrm{a}}$ \\
\hline \multirow{3}{*}{ AIP } & $\mathrm{N}$ & - & 324 & 12 & 14 \\
\hline & $\%$ & - & 92.6 & 3.4 & 4 \\
\hline & mmol..$^{-1}$ & - & $-0.26 \pm 0.17^{\circ}$ & $0.14 \pm 0.02^{b}$ & $0.40 \pm 0.17^{a}$ \\
\hline
\end{tabular}

$a, b, c, d v a l u e s$ represent statistically significant differences between groups at $p<0.05$ (Tukey test).

with regard to TC values (Table $1-3$ ). It is noteworthy that WHtR and TC values increased simultaneously, as shown in Table 2, 3. Statistically significant differences in WHtR were only observed in males according to reference values stated for TC.

Physiological levels of HDL (men $\geq 1.00 \mathrm{mmol}^{-1}{ }^{-1}$, and women $\left.\geq 1.29 \mathrm{mmol}^{-\mathrm{l}^{-1}}\right)$ were measured in 299 probands $(59.8 \%)$, including 94 men (62.7\%) and 205 women (58.6\%) (Table 1-3). Risk HDL levels (for men $<1.00 \mathrm{mmol}^{-1}$, and for women $<1.29$ mmol. $1^{-1}$ ) were observed in 201 individuals (40.2\%), including 56 men (37.3\%) and 145 women (41.4\%). When comparing statistical differences between groups according to reference values, we found statistically significant mutual relationships. An increase in HDL was observed with a decrease in WHtR in both men and women (Table 2, 3).

The average value of non-HDL for all probands was $3.22 \pm 0.85$ mmol. $1^{-1}$. In comparison to women, men had 3.6\% higher values of non-HDL (Table 2, 3). Standard non-HDL levels were observed in 397 (79.4\%) individuals; 115 men $(77.0 \%)$ and 282 women (80.6\%). 103 respondents (20.6\%) had risk non-HDL levels (i.e. higher levels), including 35 men (23.0\%) and 68 women (19.4\%). WHtR values increased with the increase in non-HDL levels, reaching statistical significance for women (Table 2, 3).

The mean LDL in the whole group was $2.78 \pm 0.77 \mathrm{mmol}^{-\mathrm{l}^{-1}}$. Physiological levels of LDL $\left(\leq 3.40\right.$ mmol. $\left.{ }^{-1}\right)$ were measured in 404 probands $(80.8 \%)$, including 119 men $(79.3 \%)$ and 285 women (81.4\%) (Table 2, 3). Increased LDL $(3.4 \leq \mathrm{x} \leq 4.11)$ was recorded in 60 subjects $(12.0 \%)$, and high levels ( $\geq 4.12)$ in 26 subjects $(5.2 \%)$. Comparing the percentages of increased risk of LDL, we found that abnormalities occurred more often in men than in women. Differences between LDL in the group of men and women were evaluated as statistically significant (Table 2,3). The WHtR values significantly increased with abnormal LDL levels (Table 2, 3). 
The average TG value was $0.98 \pm 0.58 \mathrm{mmol}^{-1}$. The values of TG were $5.8 \%$ higher in men compared to women. Levels of TG lower than 0.45 mmol..$^{-1}$ were observed in 21 respondents, 3 men $(2.0 \%)$ and 18 women $(5.2 \%)$. Physiological levels of TG were measured in 429 respondents $(85.8 \%)$. Pathologically elevated levels of TG $\left(\geq 1.7 \mathrm{mmol} . \mathrm{l}^{-1}\right)$ were observed in $21 \mathrm{men}$ $(14.0 \%)$ and 29 women (8.2\%). Differences between the groups over the individual reference values were shown to be statistically significant (Table 2, 3). WHtR values were significantly higher in women - between normal and high levels of TG. (Table 2, 3).

Out of 500 respondents, 450 had physiological levels of VLDL, including 129 males $(86.0 \%)$ with an average value of $0.40 \pm 0.13$ mmol. $1^{-1}$, and 321 women $(91.4 \%$ ) with an average VLDL level of $0.38 \pm 0.13 \mathrm{mmol}^{-1} \mathrm{l}^{-1}$ (Table 1-3). Increased values of VLDL were observed in 50 cases $(10.0 \%)$, including 21 men $(14.0 \%)$ with a mean VLDL value of $0.48 \pm 0.24 \mathrm{mmol}^{-1}$ and 29 women $(8.2 \%)$ with an average VLDL level of $0.43 \pm 0.27 \mathrm{mmol}^{-1} \mathrm{l}^{-1}$. Differences were statistically significant between men and women (Table 1). It is noteworthy that WHtR and VLDL values increased simultaneously, as shown in Tables 2 and 3.

The average value of uric acid in the whole group of respondents was $280.92 \pm 72.10 \mathrm{mmol}^{-\mathrm{l}^{-1}}$. The average values of serum uric acid in men and women were $342.19 \pm 66.87$ and $254.26 \pm 60.17$ mmol. $1^{-1}$, respectively (Table 1 ). Increased values were observed in 19 men $(12.7 \%)$ with a mean value of $458.63 \pm 50.00 \mathrm{mmol}^{-\mathrm{l}^{-1}}$ (Table 2$)$, and only 14 women $(0.04 \%)$ with a mean value of $449.64 \pm 71.06 \mathrm{mmol}^{-1} \mathrm{l}^{-1}$ (Table 3). The variances between men and women were statistically significant at $\mathrm{p}<0.001$.

Average values of fasting glucose in men and women (Table 1) were approximately the same and are within the normal range. While only $1.71 \%$ of women were found with high blood glucose (Table 3), the frequency of male subjects with limit-exceeding glucose levels was up to $6 \%$ (Table 2). While the correlation relationship between glucose and WHtR was not detected, levels of uric acid (UA) correlated significantly with WHtR (Table 4).

The average values of AT1 in the studied population were 2.26 \pm 0.90 . A statistically significant $(\mathrm{p}<0.001)$ higher ratio of LDL/ HDL was observed in men when compared to women. Values of AT1 lower than 3.5, considered as normal, were observed in 442 young people, including 117 men and 325 women. More men
(23.3\%) than women (7.1\%) had abnormalities in AT1 (Table 2, 3 ). The differences in the values of AT1 between groups (men, women, all respondents) were significant. In the group with normal values, we found that women had a significantly lower ratio of AT1 in contrast to men. The WHtR values significantly increased along with the AT1 risk (Table 1-3).

Recently, the most significant measurement has been the atherogenic index of plasma (AIP) based on the values of TAG and HDL, although it is not yet widely used in clinical practice. The average value of AIP in the studied population was $-0.12 \pm$ 0.23 (Table 1-3). A reference range of $\operatorname{AIP}(<0.11)$ was observed in $88 \%$ cases. Increased risk AIP $(0.11 \leq \mathrm{x}<0.21)$ as well as high risk AIP $(\geq 0.21)$ occurred more in men. In all groups, we observed statistically significant differences in the AIP parameter. We found significantly elevated WHtR coinciding with the increasing AIP (Table 2, 3).

\section{DISCUSSION}

In our study, we observed a relationship between WHtR and atherogenic lipid parameters in a sample of young people, mostly students of Pavol Jozef Šafárik University in Košice. According to statistics, young people are the least vulnerable group at risk of developing various diseases and injuries. Nevertheless, while they are healthy, young, educated and adaptable, they are still not safe from many of the negative effects arising from consumer lifestyle. Characterization of cardio-metabolic risk factors particularly in young population may further improve the ability to predict possible future risk and then to initiate prevention, albeit delayed, or even clinical intervention.

Modifiable cardio-metabolic risk factors of lifestyle include tobacco use, physical inactivity, and a cluster of interrelated disturbances of vascular and metabolic function that have been referred to collectively as metabolic syndrome (19).

Fruchart et al. (20) mentioned that there is still no universal agreement on the exact placement of the various cardiovascular risk factors and risk markers within the old-new suggested classification. The version of Framingham risk point scores adopted by the U.S. National Cholesterol Education Program (NCEP)

Table 4. The correlation relationships between WHtR and the most widely used clinical lipid and atherogenic parameters

\begin{tabular}{|c|c|c|c|}
\hline Parameter & Pearson's coefficient $R$ & $p$ & $\begin{array}{l}\text { Significance } \\
\text { (t-test of R) }\end{array}$ \\
\hline TC & 0.128 & 0.037 & * \\
\hline TG & 0.414 & $<0.001$ & $* * *$ \\
\hline $\mathrm{HDL}$ & -0.128 & 0.037 & * \\
\hline LDL & 0.159 & 0.006 & ** \\
\hline VLDL & 0.414 & $<0.001$ & $* * *$ \\
\hline Non-HDL & 0.277 & $<0.001$ & $* * *$ \\
\hline UA & 0.204 & 0.017 & ** \\
\hline Glucose & 0.061 & 0.569 & ns \\
\hline AT1 & 0.315 & $<0.001$ & $* * \star$ \\
\hline AIP & 0.476 & $<0.001$ & $* * *$ \\
\hline
\end{tabular}

${ }^{*} p<0.05,{ }^{* *} p<0.01,{ }^{* * *} p<0.001$, ns - not significant 
Expert Panel on Detection, Evaluation, and Treatment of High Blood Cholesterol in Adults (Adult Treatment Panel III) is based on the traditional risk factors of age, sex, TC, LDL, systolic blood pressure, and smoking (21). In contrast, the Prospective Cardiovascular Münster (PROCAM) simple scoring scheme deals with 8 risk variables: age, LDL, smoking, HDL, systolic blood pressure, family history of premature myocardial infarction, diabetes mellitus, and TG (22). We found $68.6 \%$ of men (Table 2) and $67.4 \%$ of women (Table 3 ) with normal TC levels; and $84 \%$ of men vs. $86.6 \%$ of women had normal TG values, i.e. about the same for both genders. However, differences could be found within probands in normal HDL values $(62.7 \%$ vs. $58.6 \%)$ for men and women, respectively. The role of HDL in risk estimation has been systematically re-examined using the SCORE database $(23,24)$. This work has shown that HDL can contribute substantially to risk estimation if entered as an independent variable. HDL-cholesterol modifies risk at all levels as estimated from the SCORE cholesterol charts. Furthermore, this effect is seen in both genders and in all age groups, including older women (23, 24 ). As to the risk of these parameters, $14 \%$ of men and $8.2 \%$ of women for TG, and $37.3 \%$ of men and $41.1 \%$ of women for HDL parameters are indicative of the development of metabolic syndrome and the risk of developing cardiovascular diseases in this age group (18-25 years). The Caerphillly Heart Disease Study showed TG levels as a strong indicator of cardiovascular disease (CHD) risk independent of TC and HDL-cholesterol levels (25). As for LDL particles, high levels were detected in $4.7 \%$ of men and $5.4 \%$ of women. Data from the PROCAM study showed a significant relationship between hypertriglyceridemia in CHD risk, independent of LDL- and/or HDL-cholesterol levels (22). Our observations suggest that high levels of TC, LDL, and low HDL levels are more frequent in young women. Conversely, high levels of TG were detected more frequently in men. The overall statistical evaluation of these parameters between genders, however, has not brought about statistically significant differences in the TG and LDL parameters. These were marked for gender in the values of TC and HDL (Table 1). Therefore, it appears that, in the group of young people aged between 18-25 years, high-level TG is a more common risk factor for men than for women, in whom we detected the prevalence of multiple cardio-metabolic risk factors (low HDL, high LDL and TC levels).

Regarding triglyceride-rich lipoproteins, we have yet to mention those in which altered levels affect the development of metabolic syndrome and CHD. As well established by Fruchart et al. (20), these comprise a great variety of nascent and metabolically modified lipoprotein particles. The capacity to enter the subintimal area of the vasculature is inversely related to the size of the lipid particle, whereas chylomicrons and large VLDL particles are unable to pass through the endothelial layer. Smaller VLDL, intermediate density lipoprotein (IDL) and LDL particles can enter the subintimal space where they undergo modification by means of reactive oxygen and nitrogen species produced by arterial wall cells. Data show a direct link between small VLDL/ IDL and atherogenesis. Remnant lipoproteins derived from chylomicrons and/or VLDL have also been shown to promote atherogenesis. We found that $86 \%$ of men and $91.4 \%$ of women had normal values of VLDL, which also means that higher levels of VLDL were detected more often in men than in women $(14 \%$ and $8.2 \%$, respectively) (Table 2,3 ).
Non-HDL is total cholesterol minus HDL, which is the sum of VLDL, IDL and LDL. Therefore, non-HDL includes the cholesterol in all atherogenic apoB-containing lipoproteins: TG-enriched lipoproteins, chylomicrons, chylomicrons remnants, VLDL and VLDL remnants, IDL, LDL, and lipoprotein. As LDL and TG confer greater risk for CHD than LDL alone, elevated levels of non-HDL predict cardiovascular disease and cardiovascular mortality even better than those of LDL (26). As shown in Tables 2 and 3 , up to $23 \%$ of men and $19.4 \%$ of women were found to have high levels of non-HDL. Among all measured TG-rich lipoprotein parameters, this indicator stood at high levels for both genders in up to approximately $20 \%$ of individuals.

Another parameter followed was serum uric acid (SUA) levels. There is considerable literature on the correlation between urate levels and many recognized cardiovascular risk factors. The relative importance of these associations remains controversial. Some experts, such as the Framingham Heart Study group, have argued that UA is not a risk factor for cardiovascular disease and that clinicians should rely on classical risk factors in patient assessment (27). However, Gagliardi et al. (28) concluded that there is strong evidence that, for most subjects, increased UA concentrations and gout are associated with subclinical atherosclerosis and increased risk of cardiovascular events, especially in the presence of metabolic syndrome. The justification for the review of this parameter for us is mainly based on knowledge of the reactivity of uric acid. In addition, with current eating habits, it is necessary to mention that uric acid synthesis is induced as a negative side effect of higher intake of added sugars (sucrose, fructose) through fructose-mediated generation of uric acid (29) followed by promotion of pro-inflammatory activities either in vascular smooth muscle and endothelial cells or by passage through the blood-brain barrier in the hypothalamus (30). It was proposed that uric acid nitration/nitrosation products may play a pivotal role in human pathophysiology by at least three distinct pathways, especially in the reactions with peroxynitrite leading to the formation of alkylating molecules (6). We found $12.7 \%$ of male and $4 \%$ of female respondents with high levels of SUA. At the same time, atherogenic index AT1 and AIP values were high in men $(23.3 \%$ and $13.4 \%)$ and less in women $(7.1 \%$ and $4 \%$ ) (Table 2, 3). In all three levels, we also found significant statistical differences between genders (Table1). Higher uric acid and low-grade inflammation are associated with impaired lipoprotein metabolism even before clinically visible symptoms of atherosclerosis are apparent (31). Furthermore, if we mention the relatively high percentage of male respondents with high levels of non-HDL, the results are again not in favour of men.

The study of Lu et al. (30) confirmed that hyperuricemia is the key mechanism of dyslipidemia and glucose intolerance. The hypothalamic inflammation mediated by uric acid is an early event in the development of metabolic abnormalities in peripheral tissue (32). Hypothalamic inflammation increases baseline plasma insulin, accompanied by impaired insulin signaling in liver and skeletal muscle $(33,34)$. Therefore, we can assume that the detected high levels of uric acid (up to $12.7 \%$ ) trigger or cause hyperglycemia detected in almost $6 \%$ of young men (Table 2). Interestingly, even though the frequency of women detected with hyperglycemia was low, at just $1.71 \%$ (Table 3 ), this also accounted for about half of those with hyperuricemia (4\%). 
Conventional anthropometric indices have poor sensitivity and specificity for detecting people with increased cardio-metabolic risks. The most common tool is the calculation of BMI, which does not take into account the increased muscle mass typical for athletes or fat distribution in the body, even though this has an impact on health and the risks associated with obesity. Therefore, physicians have come to use simple noninvasive anthropometric methods like waist circumference and its ratio to height, as studies using a tomographic technique found better correlation based on the relationship with intra-abdominal fat and its changes (35). Increased waist circumference is likely to be related to increased risk factors related to the increased accumulation of visceral fat, which may result in steatosis when fat accumulates in liver cells (36). The comparison of waist circumference to height (WHtR) more clearly shows an enlarged abdomen with small stature (37). By tracking the correlation relationships between anthropometric variables of risk of CVD, WHtR, and atherogenic and lipid parameters, we found a very strong correlation dependence with non-HDL, TG, HDL, UA, AT1 and AIP, while HDL was negatively correlated (Fig. 1 and 2). A set of respondents who did not demonstrate any increased atherogenic or lipid parameters were used to find the normal range of values for WHtR. To define the scope of normal (reference) values in young Slovak people, it was based on the calculation of the 10 th and 90th percentile, i.e. the value of the variable which falls between $10 \%$ and $90 \%$ of respondents. We would suggest WHtR values from 0.39 to 0.51 as normal for young people aged $18-25$. Based on gender, normal values were from 0.41 to 0.52 for men. Values from 0.53 to 0.63 were suggested as limiting, and $>0.63$ as high. Similarly, normal values for young women ranged from 0.38 to 0.50 . Values from 0.51 to 0.60 were limiting, and $>0.60$ as high. Small differences detected in the limiting values of WHtR between men (0.52), and women (0.50) are due to the fact that women are generally shorter (by about $8.49 \%$ )

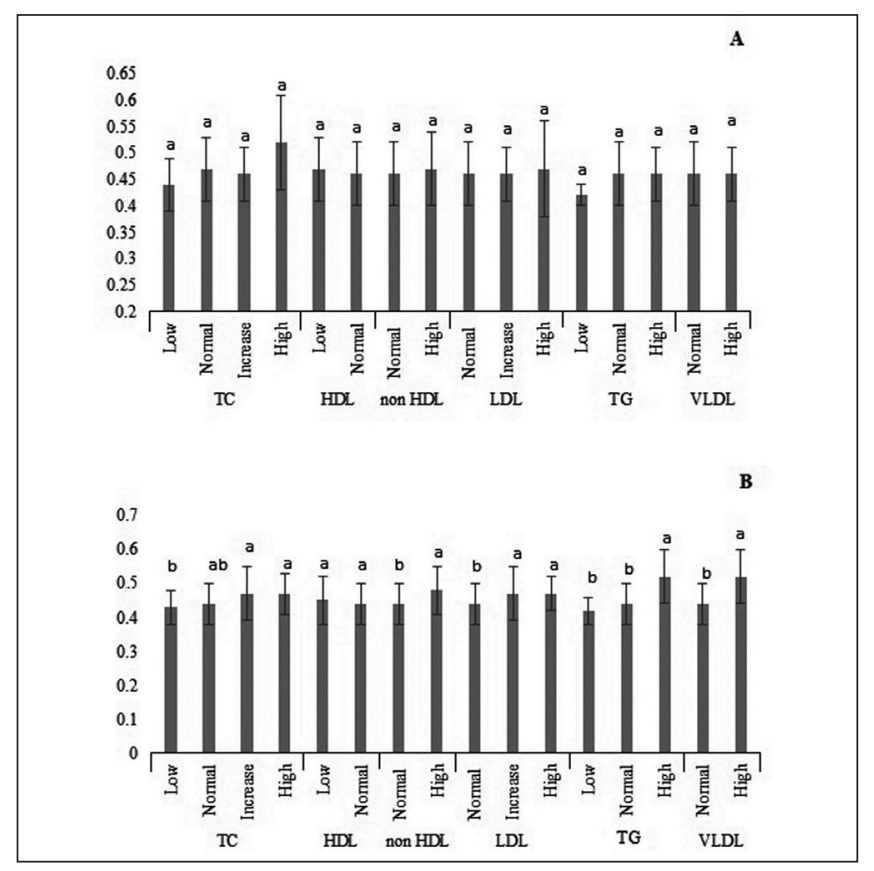

Fig. 1. Comparison of lipid parameters and WHtR in men $(A)$ and women $(B)$. Values $a, b$ represent statistically significant differences between groups (Tukey test).

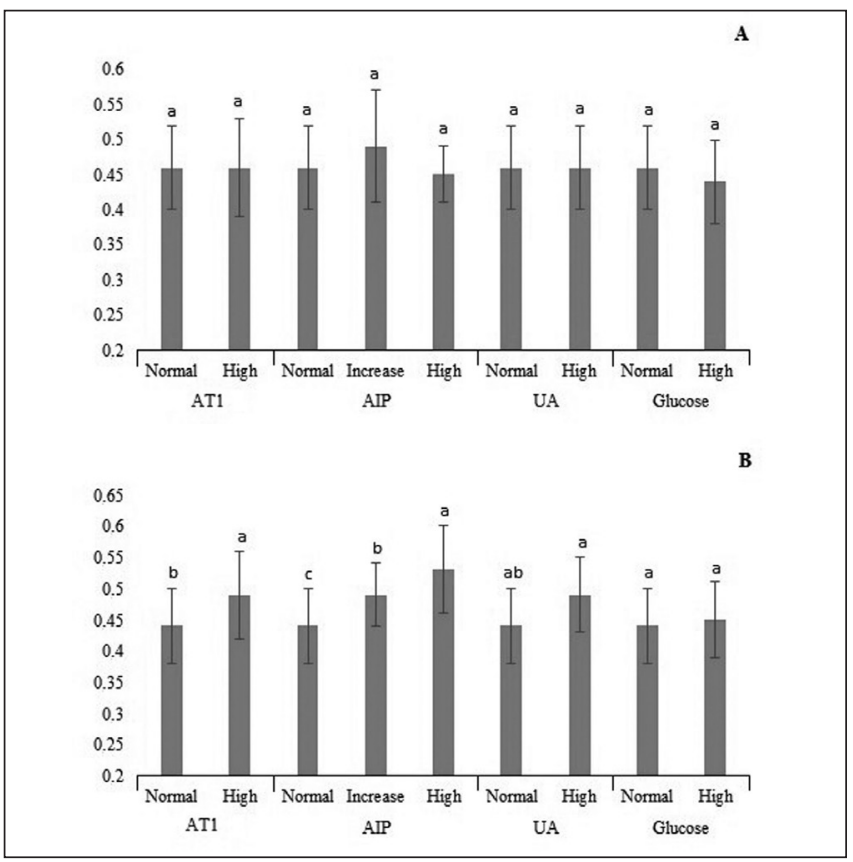

Fig. 2. Comparison of glucose, uric acid, atherogenic indices and $W H t R$ in men $(A)$ and women (B). Values $a, b$ represent statistically significant differences between groups (Tukey test).

and also have a much lower prevalence of abdominal obesity (by about $15.3 \%$ ). The values observed are consistent with the values valid for different populations (10-14).

\section{CONCLUSIONS}

This paper presents the results from blood serum analyses in 500 clinically healthy young people, mostly medical students. Values of TC, HDL, TG, calculated LDL and HDL, UA, fasting glucose, and atherogenic indicies AT1 (LDL/HDL) and AIP (log (TG/HDL)) were determined in blood serum. The aforementioned variables were compared with the simple anthropometric parameter, WHtR. We found approximately $20 \%$ of men aged between 18 and 25 showing higher prevalence of cardiometabolic risk factors. Monitoring parameters in women, with the exception of non-HDL and HDL, pointed to a lower incidence of risk factors. Despite the relatively small group of volunteers who participated in the study, the WHtR in the convenient sample of young Slovak population is a good indicator of abnormalities in the lipid profile and associated cardio-metabolic risks. The limit values for both genders tend towards 0.51 , which correspond to values from other studies.

\section{Acknowledgements}

We would like to express special thanks to medical staff (Katarína Gerová, Blanka Kozmová and Mária Grichová) who helped us secure blood collection. The study was supported by grants from the Scientific Grant Agency of the Ministry of Education, Science, Research and Sport of the Slovak Republic no. 1/1236/12, and 10/GSD/2011, 1/GSŠ/2011, 3/GSŠ/2011, 4/GSŠ/2011.

\section{Conflict of Interests}

None declared 


\section{REFERENCES}

1. Kesteloot H, Sans S, Kromhout D. Dynamics of cardiovascular and allcause mortality in Western and Eastern Europe between 1970 and 2000. Eur Heart J. 2006 Jan;27(1):107-13.

2. Müller-Nordhorn J, Binting S, Roll S, Willich SN. An update on regional variation in cardiovascular mortality within Europe. Eur Heart J. 2008 May;29(10):1316-26.

3. Hujova Z, Lesniakova M. Anthropometric risk factors of atherosclerosis: differences between urban and rural East-Slovakian children and adolescents. Bratisl Lek Listy. 2011;112(9):491-6.

4. Hujova Z, Rostakova K. Several anthropometric predictors of cardiovascular disease in central Slovakian adults: socioeconomic and educational differences. Bratisl Lek Listy. 2013;114(1):31-5.

5. Grundy SM. Gamma-glutamyl transferase: another biomarker for metabolic syndrome and cardiovascular risk. Arterioscler Thromb Vasc Biol. 2007 Jan;27(1):4-7

6. Gersch C, Palii SP, Imaram W, Kim KM, Karumanchi SA, Angerhofer A, et al. Reactions of peroxynitrite with uric acid: formation of reactive intermediates, alkylated products and triuret, and in vivo production of triuret under conditions of oxidative stress. Nucleosides Nucleotides Nucleic Acids. 2009 Feb;28(2):118-49.

7. Sebo P, Beer-Borst S, Haller DM, Bovier PA. Reliability of doctors' anthropometric measurements to detect obesity. Prev Med. 2008 Oct;47(4):389-93.

8. Mason C, Craig CL, Katzmarzyk PT. Influence of central and extremity circumferences on all-cause mortality in men and women. Obesity (Silver Spring). 2008 Dec;16(12):2690-5

9. Wang J, Thornton JC, Bari S, Williamson B, Gallagher D, Heymsfield $\mathrm{SB}$, et al. Comparisons of waist circumferences measured at 4 sites. Am J Clin Nutr. 2003 Feb;77(2):379-84.

10. Ashwell M, Gunn P, Gibson S. Waist-to-height ratio is a better screening tool than waist circumference and BMI for adult cardiometabolic risk factors: systematic review and meta-analysis. Obes Rev. 2012 Mar;13(3):275-86.

11. Browning LM, Hsieh SD, Ashwell M. A systematic review of waist-toheight ratio as a screening tool for the prediction of cardiovascular disease and diabetes: 0.5 could be a suitable global boundary value. Nutr Res Rev. 2010 Dec;23(2):247-69.

12. Jayawardana R, Ranasinghe P, Sheriff MH, Matthews DR, Katulanda P. Waist to height ratio: a better anthropometric marker of diabetes and cardio-metabolic risks in South Asian adults. Diabetes Res Clin Pract. 2013 Mar;99(3):292-9.

13. Zeng Q, He Y, Dong S, Zhao X, Chen Z, Song Z, et al. Optimal cut-off values of BMI, waist circumference and waist:height ratio for defining obesity in Chinese adults. Br J Nutr. 2014 Nov 28;112(10):1735-44.

14. Zhu Q, Shen F, Ye T, Zhou Q, Deng H, Gu X. Waist-to-height ratio is an appropriate index for identifying cardiometabolic risk in Chinese individuals with normal body mass index and waist circumference. J Diabetes. 2014 Nov;6(6):527-34.

15. Hertelyova Z, Salaj R, Chmelarova A, Dombrovsky P, Dvorakova MC, Kruzliak P. The association between lipid parameters and obesity in university students. J Endocrinol Invest. 2015 Jan 20. In Press.

16. Friedewald WT, Levy RI, Fredrickson DS. Estimation of the concentration of low-density lipoprotein cholesterol in plasma, without use of the preparative ultracentrifuge. Clin Chem. 1972 Jun;18(6):499-502.

17. Dobiásová M. Atherogenic index of plasma $[\log ($ triglycerides/HDLcholesterol)]: theoretical and practical implications. Clin Chem. 2004 Jul;50(7):1113-5

18. Dobiásová M. AIP - atherogenic index of plasma as a significant predictor of cardiovascular risk: from research to practice. Vnitr Lek. 2006 Jan;52(1):64-71. (In Czech.)

19. Cannon CP. Cardiovascular disease and modifiable cardiometabolic risk factors. Clin Cornerstone. 2007;8(3):11-28.

20. Fruchart JC, Nierman MC, Stroes ES, Kastelein JJ, Duriez P. New risk factors for atherosclerosis and patient risk assessment. Circulation. 2004 Jun 15;109(23 Suppl 1):III15-9.

21. National Cholesterol Education Program (NCEP) Expert Panel on Detection, Evaluation, and Treatment of High Blood Cholesterol in Adults
(Adult Treatment Panel III). Third Report of the National Cholesterol Education Program (NCEP) Expert Panel on Detection, Evaluation, and Treatment of High Blood Cholesterol in Adults (Adult Treatment Panel III) final report. Circulation. 2002 Dec 17;106(25):3143-421.

22. Assmann G, Cullen P, Schulte H. Simple scoring scheme for calculating the risk of acute coronary events based on the 10-year follow-up of the prospective cardiovascular Münster (PROCAM) study. Circulation. 2002 Jan 22;105(3):310-5.

23. Cooney MT, Dudina A, De Bacquer D, Fitzgerald A, Conroy R, Sans S, et al.; SCORE Investigators. How much does HDL cholesterol add to risk estimation? A report from the SCORE Investigators. Eur J Cardiovasc Prev Rehabil. 2009 Jun;16(3):304-14.

24. Cooney MT, Dudina A, De Bacquer D, Wilhelmsen L, Sans S, Menotti A, et al.; SCORE investigators. HDL cholesterol protects against cardiovascular disease in both genders, at all ages and at all levels of risk. Atherosclerosis. 2009 Oct;206(2):611-6.

25. Yarnell JW, Patterson CC, Sweetnam PM, Thomas HF, Bainton D, Elwood PC, et al. Do total and high density lipoprotein cholesterol and triglycerides act independently in the prediction of ischemic heart disease? Ten-year follow-up of Caerphilly and Speedwell Cohorts. Arterioscler Thromb Vasc Biol. 2001 Aug;21(8):1340-5.

26. Welty FK. How do elevated triglycerides and low HDL-cholesterol affect inflammation and atherothrombosis? Curr Cardiol Rep. 2013 Sep;15(9):400. doi: 10.1007/s11886-013-0400-4.

27. Gómez-Marcos MA, Recio-Rodríguez JI, Patino-Alonso MC, AgudoConde C, Rodríguez-Sánchez E, Gómez-Sánchez L, et al.; Vasorisk group. Relationship between uric acid and vascular structure and function in hypertensive patients and sex-related differences. Am J Hypertens. 2013 May;26(5):599-607.

28. Gagliardi AC, Miname MH, Santos RD. Uric acid: A marker of increased cardiovascular risk. Atherosclerosis. 2009 Jan;202(1):11-7.

29. Johnson RJ, Nakagawa T, Sanchez-Lozada LG, Shafiu M, Sundaram S, Le M, et al. Sugar, uric acid, and the etiology of diabetes and obesity. Diabetes. 2013 Oct;62(10):3307-15.

30. Lu W, Xu Y, Shao X, Gao F, Li Y, Hu J, et al. Uric Acid Produces an Inflammatory Response through Activation of NF- $\kappa$ B in the Hypothalamus: Implications for the Pathogenesis of Metabolic Disorders. Sci Rep. 2015 Jul 16;5:12144. doi: 10.1038/srep12144.

31. Vekic J, Jelic-Ivanovic Z, Spasojevic-Kalimanovska V, Memon L, Zeljkovic A, Bogavac-Stanojevic N, et al. High serum uric acid and lowgrade inflammation are associated with smaller LDL and HDL particles. Atherosclerosis. 2009 Mar;203(1):236-42.

32. Zhang X, Zhang G, Zhang H, Karin M, Bai H, Cai D. Hypothalamic IKKbeta/NF-kappaB and ER stress link overnutrition to energy imbalance and obesity. Cell. 2008 Oct 3;135(1):61-73.

33. Baldwin W, McRae S, Marek G, Wymer D, Pannu V, Baylis C, et al. Hyperuricemia as a mediator of the proinflammatory endocrine imbalance in the adipose tissue in a murine model of the metabolic syndrome. Diabetes. 2011 Apr;60(4):1258-69.

34. Arruda AP, Milanski M, Coope A, Torsoni AS, Ropelle E, Carvalho DP, et al. Low-grade hypothalamic inflammation leads to defective thermogenesis, insulin resistance, and impaired insulin secretion. Endocrinology. 2011 Apr;152(4):1314-26.

35. Ashwell M, Gunn P, Gibson S. Waist-to-height ratio is a better screening tool than waist circumference and BMI for adult cardiometabolic risk factors: systematic review and meta-analysis. Obes Rev. 2012 Mar;13(3):275-86.

36. Després JP, Lemieux S, Lamarche B, Prud'homme D, Moorjani S, Brun $\mathrm{LD}$, et al. The insulin resistance-dyslipidemic syndrome: contribution of visceral obesity and therapeutic implications. Int J Obes Relat Metab Disord. 1995 May;19 Suppl 1:S76-86.

37. Jeong SK, Seo MW, Kim YH, Kweon SS, Nam HS. Does waist indicate dyslipidemia better than BMI in Korean adult population? J Korean Med Sci. 2005 Feb;20(1):7-12. 